\title{
Comparison of Live Teaching Methods Based on the Live Classroom System and the Video Conference System in the Context of the COVID-19 epidemic: A Case Study of a Robotics Teaching Course
}

\author{
Wei-jie Yang ${ }^{1,2, *}$ and Fang-fang $\mathrm{Du}^{3}$
}

\author{
${ }^{1}$ School of Information, Guangdong Teachers College of Foreign Language and Arts, Guangzhou 510640, China \\ ${ }^{2}$ School of Information Technology in Education, South China Normal University, Guangzhou 510631, China \\ ${ }^{3}$ College of Medical Information Engineering, Guangdong Pharmaceutical University, Guangzhou 510006, China \\ *Corresponding author.Email: yangwj@gtcfla.net
}

\begin{abstract}
The live teaching based on the live classroom system (LTBLCS) and the live teaching based on the video conference system (LTBVCS) were widely applied in colleges and universities in China in the context of the COVID-19 epidemic. The purpose of this study was to compare these two live teaching methods with paired sample analysis by a case study in a robotics teaching course. Two methods of the live teaching were applied in two different phases of this course, and then surveyed each phase by using a self-designed questionnaire. Results indicate that there are significant differences in the Learning environment requirements, Learning materials and resource delivery, and Learning activity organization between the live teaching based on the live classroom system and the live teaching based on the video conference system. The factors include Learning environment requirements, Learning materials and resource delivery of LTBLCS were better than that of LTBVCS, but the capability of Learning activity organization is poorer than that of LTBVCS. However, there were no significant differences in the factors include Teacher-student interaction, Teacher's question answering and tutoring, Learning anxiety, Self-evaluation of learning performance. On this basis, some suggestions were proposed to improve live teaching.
\end{abstract}

Keywords: COVID-19 epidemic, live teaching methods, live classroom system, video conference system,

comparison

\section{INTRODUCTION}

In the spring of 2020, the sudden outbreak of the COVID19 epidemic brought new challenges to teachers and students in China. Because the Novel coronavirus pneumonia is highly infectious, fatal, and dangerous, aiming to avoid large-scale infection between teachers and students in colleges and universities, the Ministry of education requires colleges and universities across the country to implement large-scale online learning to combat with the epidemic. According to an official report by $\mathrm{Wu}$ Yan, director of the Higher Education Department of the Ministry of education of China, as of May 8, 1454 colleges and universities across the country had carried out online teaching, and 1.03 million teachers had opened 1.07 million courses online, a total of 12.26 million times. There are 17.75 million colleges and universities, about 2.3 billion students taking part in online learning [1]. Huge funds were invested by the government and universities to increase the network bandwidth, improve the performance of computing services, and other ways to improve the information technology infrastructure. As a supplement to the schools' e-learning systems, many enterprises have opened free online teaching platforms and conference live systems for teachers and students. For example, Tencent, Alibaba, Huawei, and other large hightech enterprises have opened the teaching and learning service tools based on the network community for free. Teachers in colleges and universities have changed their teaching models by using various online teaching platforms, such as MOOC and SPOC, video and broadcast, live teaching, online question answering, and counseling, etc.

In the context of the COVID-19 epidemic, live teaching has been widely used and become one of the most important online teaching methods. A survey in Guangdong Teachers College of Foreign Language and Arts showed that $62.7 \%$ of 249 teachers gave guidance by using live teaching, $77.9 \%$ of them use live teaching based on the live classroom system (LTBLCS) and $43.5 \%$ of them use live teaching based on the video conference system (LTBVCS). In online learning, students' independent learning, learner-centered instructional design, and teaching management are emphasized. What is the difference between the two kinds of live teaching methods? 
Which kind of live teaching method is more suitable for the course that integrated theory and practice? This study takes a robotics teaching course in a college as an example to make a comparative study of the above problems.

\section{LITERATURE REVIEW}

\subsection{The Live teaching}

The live teaching can be defined as a real-time teaching method for online education by using video, audio and other media under the condition of Internet. The live teaching has the characteristics of timeliness, realness, high sense of presence, interaction, and personalization. The live teaching originated from the traditional course based on video teaching. Early online distance education mainly relied on the courses based on Web pages and video recordings, and students carried out autonomous learning in distance [2]. The traditional form of distance education has great defects in teacher-student interaction, teaching presence, and learning atmosphere. Therefore, video live conference was used to improve teaching. The teaching method based on video conference system was different from the recorded video lectures; video conference is a real-time way of distance education between teachers and learners. Teachers and students can interact with each other in dual-way communication via video conference system [3]. Live education based on video conference requires teachers and students to access the network conference system at the same time [4], which is different from the traditional idea of distance education which emphasis on not limited by time and place, and the platform construction cost and use cost of video conference is relatively high. Since 2016, with the maturity of mobile network technology, the improvement of mobile terminal performance, and the rapid development of social media, mobile-based live broadcasting technology has been rising in the field of Internet application, from live broadcasting, game live broadcasting, and entertainment live broadcasting to other areas, and gradually penetrated all aspects of human learning, life, and work. With the emergence of zero thresholds like live broadcasting platforms, live broadcasting technology began to enter the blowout period. At the same time, many high-tech companies and educational training institutions in China have invested in the research and development of different live video teaching and audio teaching platforms, such as Tencent Classroom, Zoom, CCtalk of hujiang.com, Billibili's live classroom. Many training institutions including xueersi.com, sanhao.com, and dingdang.com have vigorously promoted live education. In the context of the epidemic, the online live classroom has entered into the daily teaching of popularization from being specially used for academic lectures, meetings, and online training.

\subsection{Live teaching based on the live classroom system}

Live teaching based on the live classroom system (LTBLCS) refers to a live online teaching method based on the live classroom system. The system usually integrated live teaching, real-time recording, and broadcasting, classroom hands-up, teaching resources delivery, student management, practices, time statistics, and other functions in the client program[5] [6]. With the support of cloud computing technology, dozens to thousands of people can learn synchronously at different places. Compared with the traditional live network system or video conference system, the live classroom system is much lighter. The former usually need the equipment or resources like a special professional camera, video studio and live broadcast software, photography, and even need the support from an editing team, while the latter only need a PC or tablet computer or a Pad with a camera and microphone. Even if the teacher can access the network with a smart mobile phone, LTBLCS can be carried out. However, LTBLCS also has some limitations in technology. Most of the record and broadcast functions of the system were realized by recording the screen and audio from the teacher's terminal and then compose them synchronously, and the screen and audio from the students' terminal can't be recorded. Generally, the functions of the students' client were much less. For example, Tencent classroom system used in this study does not support audio and video recording on the student side. Students mainly participate in learning interaction by using hands up, publishing text, and uploading resources in chat windows.

\subsection{Live teaching based on the video conference system}

Live teaching based on the video conference system (LTBVCS) refers to a live online teaching method based on the video conference system. The video conference system usually has multi-users real-time video and audio communication function. It provides a teaching mode by hosting a meeting for participants, and the main lecturer can deliver the learning materials and contents by sharing desktop screen, PPT, camera, or playing audio and video records. It also can share files, facilitate real-time multiperson interaction, and conduct text-based interaction in the chat window [7]. The video conference systems usually connect the real-time video and audio from multiple terminals to the server in cloud and play the selected channel in real time, so these systems usually do not support the recording and playing function, and the number of participants generally limite to 200 or less because of the processing capability of the system and bandwidth of network. And the system overload will lead to the abnormal situation of network jam or video playing intermittently. Tencent video conference system was used in this study, which has the largest number of users in China. The literature review showed that recent researches 
relative to live teaching mainly focused on the comparison on live teaching and traditional distance education, teaching models, and effects of the live teaching based on the live classroom system or video conference system. However, comparative research on the two live teaching methods is relatively rare. Analyzing the differences, advantages, and disadvantages of these two live teaching methods will lead to a deeper understanding of these two methods, and a better combination method for different courses, to provide effective guidance for the students in and after the epidemic period.

\section{METHODOLOGY}

\subsection{Research tools}

A self-designed questionnaire based on Jonathan's constructivism learning environment design theory [8] was used in this study. The questionnaire composed of student' s background information and seven factors affecting the live teaching method: Learning environment requirements (LER), Learning materials and resource delivery (LMRD), Learning activity organization (LAO), Teacher-student interaction (TSI), Teacher's question answering and tutoring (TQAT), Learning anxiety (LA), Self-evaluation of learning performance (SELP).

\subsection{Research procedure}

The course "Robotics Teaching Based on Scratch" was selected for the research case for this study. This course was a practical training course for training robotics teachers in primary schools in the modern educational technology major of Guangdong Teachers College of Foreign Language and Arts. Teachers in and outside the college were invited to complete the course together. The main contents of this course include: learn scripts of Scratch, design Scratch games, complete the programming of "ChengXiaoBen" robot with Scratch in the mblock coding editor. Before the outbreak of the epidemic, this course was to be completed in the robotics training room, and it can only be completed by the way of live teaching \& online course self-study in the period of epidemic. The reasons for choosing this course as a case study to compare two kinds of live teaching methods were as follows:(1)this course includes theoretical teaching and hands-on practice, which need to use different live teaching methods; (2)the teaching environment have changed in the context of the epidemic, and students have in-depth experience in live teaching; (3) the diversity of teaching activities was relatively rich in this course, students were require to carry out group learning and report the experimental results, so students and teachers interacted frequently during the course.

\subsection{Collections of research data}

The course "Robotics Teaching Based on Scratch" has a total of three weeks, a total of 30 class hours. 35 students participated in the course. The teacher told the students about the design and goal of this study and got their consent and cooperation. In the first and second weeks of the course, the theoretical learning and editing practice of the Scratch language programming of the robotics course was completed. The live teaching method based on classroom live teaching system was applied in this phase, and then collected the first round of data by using "WXJ" network questionnaire system. In the third week, students mainly used Scratch language to complete the project of coding with the robot, and carried out teaching report demonstration and then teacher commented on the students' performance. The live teaching method based on video conference system was applied in this phase. After the reports were completed, the second round of data was collected by using the same online questionnaire system. The content of the questionnaire used was same, only modified the expression of teaching methods accordingly. For example, in the first round of questionnaire, "the performance of my computer terminal or mobile phone can support live learning based on the live classroom system (Tencent course)", the second round of questionnaire was changed to "the performance of my computer terminal or mobile phone can support live learning based on the video conference system (Tencent conference system)". Students were required to fill in the student number when answering the questionnaire, to facilitate the comparative analysis of paired samples.

\subsection{Data analysis}

All the data were analyzed with SPSS 20. There were 34 valid samples, because one student's data was found missing during the process of data checking. To facilitate the report of statistical results of Paired Samples Statistics, the author added " 1 " and " 2 " after the factors' name which stand for LTBLCS and LTBVCS, for example, "LER1" means "learning environment requirements of live teaching based on the live classroom system", and " LER2" means "learning environment requirements of live teaching based on the video conference system", and so on. In the process of data analysis, the methods of descriptive analysis and paired samples test were used in this study.

\section{RESULTS}

\subsection{Overall comparison of two live teaching methods}

The Paired Samples Statistics results were demonstrated in Table 1 and Table 2. The mean value of LER1 (3.72) was higher than that of LER2 (3.3), indicated that the factor 
"Learning environment requirements" (LER) for LTBLCS can be easier to be met than the LTBVCS, and the $\mathrm{p}=.002$, $\mathrm{p}<.05$, indicated that there was a significant difference between the Learning environment requirements for LTBLCS and LTBVCS. The mean value of LMRD1 (4.04) was higher than that of LMRD2 (3.72), indicating that the factor "Learning materials and resource delivery" (LMRD) for LTBLCS was better than the LTBVCS, and the $\mathrm{p}=.013$, $\mathrm{p}<.05$, indicated that there was a significant difference between the capability of Learning materials and resource delivery for LTBLCS and LTBVCS. The mean value of LAO1 (3.73) was lower than that of LAO2 (3.96), indicating that the factor "Learning activity organization" (LAO) for LTBLCS was worse than the LTBVCS, and the $\mathrm{p}=.017, \mathrm{p}<.05$, indicated that there was a significant difference between the capability of Learning activity organization for LTBLCS and LTBVCS. The mean value of TSI1 (3.75) was higher than that of TSI2 (3.64), indicating that the factor "Teacher-student interaction" (TSI) for LTBLCS was a little better than the LTBVCS, and the $\mathrm{p}=.325, \mathrm{p}^{>} .05$, indicated that there was no significant difference between the Teacher-student interaction for LTBLCS and LTBVCS. The mean value of TQAT1 (3.94) was nearly equal with that of TQAT2 (3.93), $\mathrm{p}=.925$, indicated that the factor "Teacher's

question answering and tutoring” (TQAT) for LTBLCS was quite a little difference than the LTBVCS. The mean value of LA1 (3.39) was a little higher than that of LA2 (3.27), $\mathrm{p}=.274$, indicated that the level of Learning anxiety (LA) for LTBLCS was a little higher than the LTBVCS, but no significant. The mean value of SELP1 (3.65) was a little lower than that of SELP2 (3.75), $p=.185$, indicated that the students' Self-evaluation of learning performance (SELP) for LTBLCS was a little lower than the LTBVCS, but no significant.

Table 1. Paired Samples Statistics

\begin{tabular}{c|c|c|c|c}
\hline \multicolumn{2}{|c|}{ Pair } & Mean & $\mathbf{N}$ & Std. Deviation \\
\hline \multirow{2}{*}{ Pair 1 } & LER1 & 3.7157 & 34 & .63634 \\
\cline { 2 - 5 } & LER2 & 3.3039 & 34 & .89280 \\
\hline \multirow{2}{*}{ Pair 2 } & LMRD1 & 4.0441 & 34 & .69759 \\
\cline { 2 - 5 } & LMRD2 & 3.7206 & 34 & .71447 \\
\hline \multirow{2}{*}{ Pair 3 } & LAO1 & 3.7255 & 34 & .48179 \\
\cline { 2 - 5 } & LAO2 & 3.9608 & 34 & .66548 \\
\hline \multirow{2}{*}{ Pair 4 } & TSI1 & 3.7549 & 34 & .67324 \\
\cline { 2 - 5 } & TSI2 & 3.6373 & 34 & .73107 \\
\hline \multirow{2}{*}{ Pair 5 } & TAS1 & 3.9412 & 34 & .62749 \\
\cline { 2 - 5 } & TQAT2 & 3.9314 & 34 & .70962 \\
\hline \multirow{2}{*}{ Pair 6 } & LA1 & 3.3897 & 34 & .68587 \\
\cline { 2 - 5 } & LA2 & 3.2721 & 34 & .82885 \\
\hline \multirow{2}{*}{ Pair 7 } & SELP1 & 3.6471 & 34 & .64584 \\
\cline { 2 - 5 } & SELP2 & 3.7451 & 34 & .58060 \\
\hline
\end{tabular}

Table 2. Paired Samples Test

\begin{tabular}{|c|c|c|c|c|c|c|c|c|c|}
\hline & \multirow{3}{*}{ Pair } & \multicolumn{5}{|c|}{ Paired Differences } & \multirow{3}{*}{$\mathbf{t}$} & \multirow{3}{*}{ df } & \multirow{3}{*}{$\begin{array}{l}\text { Sig. (2- } \\
\text { tailLER) }\end{array}$} \\
\hline & & \multirow{2}{*}{ Mean } & \multirow{2}{*}{ Std. Deviation } & \multirow{2}{*}{ Std. Error Mean } & \multicolumn{2}{|c|}{ 95\% Confidence Interval of the Difference } & & & \\
\hline & & & & & Lower & Upper & & & \\
\hline$\overline{\text { Pair } 1}$ & LER1 - LER2 & .41176 & .69660 & .11947 & .16871 & .65482 & 3.447 & 33 & .002 \\
\hline$\overline{\text { Pair } 2}$ & LMRD1 - LMRD2 & .32353 & .71650 & .12288 & .07353 & .57353 & 2.633 & 33 & .013 \\
\hline Pair 3 & LAO1 - LAO2 & -.23529 & .54760 & .09391 & -.42636 & -.04423 & -2.505 & 33 & .017 \\
\hline$\overline{\text { Pair } 4}$ & TSI1 - TSI2 & .11765 & 68599 & .11765 & -.12171 & .35700 & 1.000 & 33 & .325 \\
\hline$\overline{\text { Pair } 5}$ & TQAT1 - TQAT2 & .00980 & .60573 & .10388 & -.20154 & .22115 & .094 & 33 & .925 \\
\hline$\overline{\text { Pair } 6}$ & LA1 - LA2 & .11765 & .61618 & .10567 & -.09735 & .33264 & 1.113 & 33 & .274 \\
\hline Pair 7 & SELP1 - SELP2 & -.09804 & .42267 & .07249 & -.24552 & .04944 & -1.353 & 33 & .185 \\
\hline
\end{tabular}

\subsection{Details of the differences between the two live teaching method}

The results show that there were significant differences between the two methods in factors: LEM, LMRD, and LAO. In order to make clear the differences in the internal items of each factor, the author made further comparative analysis of the sub-items of the three factors.

Differences in learning environment requirements. For the question "My computer terminal or mobile phone can support the live teaching (LTBLCS/LTBVCS)",the mean value of LTBLCS(4.18) was higher than that of LTBVCS(3.76), and the Paired Samples Test result $p=.003$, indicated that there was a significant difference between students' terminal requirements in LTBLCS and LTBVCS, and can more easily meet in the LTBLCS. For the question "My Network Broadband and Access Conditions can support the live teaching (LTBLCS/LTBVCS)", the mean value of LTBLCS(3.47) was higher than that of LTBVCS(3.12), and the Paired Samples Test result $p=.003$, indicated that there was a significant difference between students' network requirements in LTBLCS and LTBVCS, and the network requirements in LTBLCS was relatively low. For the question "My Learning environment (learning place, camera conditions, sound, light, etc.) can support the live teaching (LTBLCS/LTBVCS)", the mean value of LTBLCS(3.50) was higher than that of LTBVCS(3.03), and the Paired Samples Test result $\mathrm{p}=.003$, indicated that there was a significant difference between students' 
Learning environment in LTBLCS and LTBVCS, and the Learning environment requirement in LTBLCS was relatively low.

Differences in learning materials and resource delivery. For the question "In the live teaching (LTBLCS/LTBVCS), I can see the PPT and screen sharing content published by teachers", the mean value of LTBLCS (4.03) was higher than that of LTBVCS (3.74), and the Paired Samples Test result $\mathrm{p}=.023$, indicated that there was a significant difference between whether students can see the content clearly in LTBLCS and LTBVCS, and the LTBLCS was better. For the question "In the live teaching(LTBLCS/LTBVCS), I can see the teacher's theory teaching, operation steps for software and hardware", the mean value of LTBLCS(3.82) was higher than that of LTBVCS(3.82), and the Paired Samples Test result $\mathrm{p}=1.000$, indicated that there was no significant difference between LTBLCS and LTBVCS. For the question "In the live teaching (LTBLCS/LTBVCS), I can access to learning resources provided by teachers or students conveniently", the mean value of LTBLCS (4.12) was higher than that of LTBVCS (3.79), and the Paired Samples Test result $\mathrm{p}=.019$, indicated that there was a significant difference between whether students can access to learning resources conveniently in LTBLCS and LTBVCS, and the LTBLCS was better. For the question "In the live teaching (LTBLCS/LTBVCS), I can view the teaching recording and broadcasting content in the playback video conveniently", the mean value of LTBLCS (4.21) was higher than that of LTBVCS (3.53), and the Paired Samples Test result $p=.006$, indicated that there was a significant difference between whether students can view the playback video conveniently in LTBLCS and LTBVCS, and the LTBLCS was better.

Differences in learning activity organization. For the question "In the live teaching (LTBLCS/LTBVCS), teachers can easily organize us to carry out group learning, display or group discussion", the mean value of LTBLCS (3.68) was lower than that of LTBVCS (4.15), and the Paired Samples Test result $p=.001$, indicated that there was a significant difference between whether teacher can easily organize group activities in LTBLCS and LTBVCS, and the LTBVCS was much better. For the question "In the live teaching (LTBLCS/LTBVCS), teachers can easily invite outside experts or business mentors to give us lectures", the mean value of LTBLCS (3.65) was lower than that of LTBVCS (4.00), and the Paired Samples Test result $\mathrm{p}=.001$, indicated that there was a significant difference between whether teacher can invite outer experts for classroom in LTBLCS and LTBVCS, and the LTBVCS was much better. For the question "In the live teaching (LTBLCS/LTBVCS), teachers can easily make a roll call and other teaching management work", the mean value of LTBLCS (3.85) was higher than that of LTBVCS (3.74), and the Paired Samples Test result $p=.325$, indicated that there was no significant difference between whether teacher can do management work in LTBLCS and LTBVCS, and the LTBLCS was a little better.

\section{DISCUSSION}

Teachers and students should rationally understand the functional differences between different live teaching systems, and adopt the appropriate mixed live broadcast method in live teaching. As is shown in the results, use the LTBLCS in the theoretical teaching part, is conducive to reducing the terminal and network requirements, enabling students to see the theoretical knowledge content, as well as the procedures for specific operation steps. And students can regain the teaching information by looking back at videos when they missed the live teaching. And LTBVCS should be used in the project practice stage and student achievement report stage, so as to give the initiative of learning back to the students. Generally, teachers should rationally recognize the limitations of the two kinds of live teaching methods, choose the appropriate live teaching method according to different teaching phases, and different teaching tasks, and it is better to adopt the mixed live teaching method in a course.

In the live teaching based on the live classroom system, teachers should pay attention to the elaborate teaching design and optimize the activity organization. Teachers should emphasis on improving the level of interaction between teachers and students, increasing the design of learning activities and control the time reasonably. The research data showed that the LTBLCS is more limited in the form of interactive activities, weaker in group learning and corroborate activities. Because the teacher can't see the students' body and eye responses in the LTBLCS, it's easy to become the teacher's "monologue" during the course implementation. So teachers need to add interactive activities, such as adding questions in the chat room, using the option of numbers for answers, to facilitate students' answers, or let students send expression packs and other ways to inspire the teaching atmosphere.

In the live teaching based on the video conference system, teachers should pay attention to the etiquette and ceremony of teaching, and provide additional learning resources. Because the video conference system will combine multiple real-time videos and voices of teachers and students at the same time, it is convenient to carry out interaction between teachers and students, to make personal theme speech or chat together at the same time. There are higher requirements for the light, a quieter environment, a higher network speed for videobroadcasting. If the light is too dark or too noisy, or the students forget to turn off the microphone, that will interfere with the live teaching order. So pay attention to the etiquette of teaching, and ask the students to dress properly will form a good habit of online teaching. In the process of live teaching, teachers should also cultivate and enhance the sense of teaching ceremony by greeting students and saying goodbye. Because the conference system doesn't provide playbacks for teaching, so teachers need to provide learning materials through online courses or other resources.

In both methods of live teaching, teachers should increase teachers' learning support, reduce students' learning anxiety and improve the learning performance. During the 
epidemic, students can't return to school for a long time, they can't get printed materials, can't use equipment to complete necessary experiments, and some students even don't have a computer at home and can't access network. Therefore, it is necessary to improve the teaching efficiency by increasing the learning support, including timely answering questions, effective homework comments, inspections, and responding to common problems in time. In the epidemic period, under the combined effects of ICT technology difficulties, low sense of academic achievement anxiety and non-compulsory learning, students have more learning burnout problems, so teachers should pay more attention to students and use more group learning methods to improve students' learning performance. Teachers need to concern about the students' attendance, participation time, and homework completion and interaction level in both live teaching methods.

\section{CONCLUSION}

There were significant differences in the Learning environment requirements, Learning materials and resource delivery, and Learning activity organization between the live teaching methods based on live classroom system (LTBLCS) and live teaching based on the video conference system (LTBVCS). The factors include Learning environment requirements, Learning materials and resource delivery of LTBLCS were better than that of LTBVCS, but the capability of Learning activity organization is poorer than that of LTBVCS. However, there were no significant differences in the factors included Teacher-student interaction, Teacher's question answering and tutoring, Learning anxiety, Self-evaluation of learning performance. The process of live teaching is not only affected by the functional differences and characteristics of the system platform or software, but also related to teachers' instructional design, teachers-students interaction and learning management. Therefore, it is necessary for teachers to adopt appropriate mixed live teaching methods, improve and optimize the design of teaching and learning activities, pay more attention to teaching etiquette and sense of teaching ceremony, provide good learning resources, and increase supporting and reduce the level of learning anxiety, to improve the effectiveness of live teaching and students' learning performance.

\section{ACKNOWLEDGMENT}

This study was supported by the Major Project of the National Social Science Fund (Grant \#18ZDA334 in 2018), Major Scientific Research Projects of Universities in Guangdong Province (Grant \#2017GWTSCX003 in 2018), International education Demonstration Area Construction Research Project in Guangdong-Hong Kong-Macao Greater Bay Area (Grant \#2020WQYB034 in 2020), Funded Project of Guangdong Teachers College of Foreign Language and Arts(Grant \#2018ZX01 in 2018).

\section{REFERENCES}

[1] Ministry of Education: 1454 colleges and universities nationwide carry out online teaching for 17.75 million college students.[online]Available at<http://www.edu.cn/xxh/focus/xs_hui_yi/202005/t202 00514_1727220.shtml >(18/04/2020 08:47)

[2]Du Qiong-Ying. Approach to autonomous learning in modern distance education. Distance education in China, 000(005) (2002) 19-21.

[3] Walsh, J., \& Reese, B. Distance learning's growing reach. THE Journal, 22(6) (1995), 58-62.

[4]Chen, YauJane, \& Willits, Fern K.. A path analysis of the concepts in Moore's theory of transactional distance in a videoconferencing learning environment. journal of distance education, 13(2) (2007) 748-755.

[5] Tencent Classroom Client (2020). [online]Available at $<$ https://ke.qq.com/download/pcapp.html >(18/05/202 0 08:47)

[6] CCTalk Client (2020). [online]Available at $<$ https://www.cctalk.com/download >(18/05/2020 09:47)

[7] Tencent video conference system (2020).[online]Available at $<$ https://cloud.tencent.com/act/event/tencentmeeting_f ree $>(18 / 05 / 2020$ 09:47)

[8] Jonassen, D. H., \& Rohrer-Murphy, L. Activity theory as a framework for designing constructivist learning environments. Educational Technology Research and Development, 47(1) (1999) 61-79. 